\title{
Research Paper: Attitude Assessment of Managers and Staffs About Urban Modification for People With Disabilities in Municipality of Tehran City
}

\author{
Seyedeh Nafiseh Askarinejad ${ }^{1}$, Kiyanoosh Abdi' ${ }^{1}$ Enayatollah Bakhshi ${ }^{2}$, Mehdi Amiri ${ }^{3},{ }^{*}$ Asghar Makarem ${ }^{1}$
}

1. Department of Rehabilitation Management, University of Social Welfare and Rehabilitation Sciences, Tehran, Iran.

2. Department of Biostatistics, University of Social Welfare and Rehabilitation Sciences, Tehran, Iran.

3. Administration of Health, Deputy of Social and Cultural Affairs, Municipality, Tehran, Iran.

Citation: Askarinejad SN, Abdi K, Bakhshi E, Amiri M, Makarem A. [Attitude Assessment of Managers and Staffs About Urban Modification for People With Disabilities in Municipality of Tehran City (Persian)]. Archives of Rehabilitation. 2017; 18(1):33-42. https://doi.org/10.21859/jrehab-180133

https://doi.org/10.21859/jrehab-180133

Received: 25 Nov. 2016 Accepted: 19 Feb. 2017

Keywords: Attitude assessment, Staffs, People with disabilities, Modification, Municipality

\section{ABSTRACT}

Objective According to a report published by the World Health Organization in $2011,15 \%$ of the world's population is disabled, which sums up to more than a billion people. People with disabilities comprise the largest group of people in Asia. Communities can facilitate the participation of people with disabilities in society by creating an atmosphere that is appropriate for them, an activity which involves the urban modification of the environment. Therefore, this study aimed at determining the attitude of managers and staff with respect to urban modification for people afflicted with disabilities in the municipality of Tehran city.

Methods \& Materials A cross-sectional, descriptive - analytic study that was conducted by cross - sectional method, that was used with the help of a valid and reliable questionnaire "Assessment of attitude of managers and staffs in about urban modification for people with disabilities". The study population comprised all managers and staffs who were working with urban modification in the municipality of Tehran City. Questionnaires were distributed among 16 managers and experts working in the modification staff of the municipality of Tehran. A total of 162 people working in one of the deputies, were selected by census to participate in the study. This study measured attitude of mangers and staffs were working in the municipality of Tehran, towards urban modification for people with disabilities in Tehran city. The Levene test was used to determine for the equality of variances. To calculate whether there is a statistically significant difference between the means of the two groups, the independent t-test was used carried out. For a comparison between two groups and ANOVA was also used to compare between the groups. Data analyzed by using The findings were evaluated using Software SPSS22.

Results The findings showed that the attitude of 17 (6.9\%) mangers and staffs regarding urban modification for people with disabilities was at the intermediate level. The attitude of 112 persons (9.62\%) was at high levels and that of 49 persons (27.5\%) was at a very good level. The average score of attitudes of manager and staffs who were familiar with disabilities was higher than those who were unfamiliar with disabilities. In addition, the average attitude score of women was better than that of men. The mean score of the attitude of people aged 55 years and older, having a Master's degree and above, and those having 12-17 years of work experience was higher than others. No significant difference was found between the groups with respect to gender $(P=0.37)$ and responsibility $(P=0.37)$. However, there was a significant difference between the two groups with respect to familiarity with disabilities $(P=0.01)$ and education $(P=0.001)$.

Conclusion This study showed that there is a significant difference in the attitude of managers and staff of municipality regarding urban modification. Recruiting people with higher educational qualifications and awareness or familiarity with the phenomenon of disability could help in the process of urban modification in the community. It is recommended to utilize the findings of this study to formulate urban modification programs across communities for the benefit of the disabled.

\section{* Corresponding Author:

Address: Department of Rehabilitation Management, University of Social Welfare and Rehabilitation Sciences, Tehran, Iran.

Tel: +98 (21) 22180132

E-Mail: amakarem2001@yahoo.com 


\title{
نكَرشسنجى از مديران و كاركنان نسبت به مناسبسازى شهرى براى افراد داراى ناتوانى در شهردارى شهر تهران سال سوسئ مدران
}

\author{
سيدهنفيسه عسكرىنزاد'، كيانوش عبدى'، عنايتالله بخشى'؛ مهدى اميرى"، "اصغر مكارم' \\ 1- كروه مديريت توانبخشى، دانشكاه علوم بهزيستى و توانبخشى، تهران، ايران.

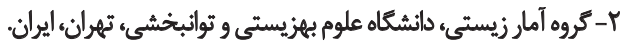

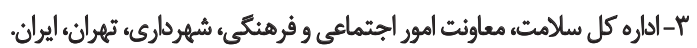

\begin{abstract}
حكبد

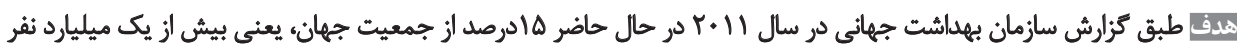

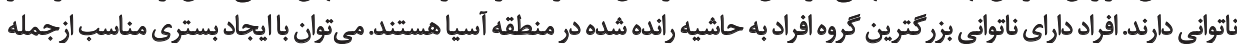

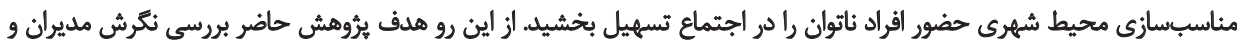

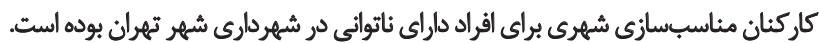

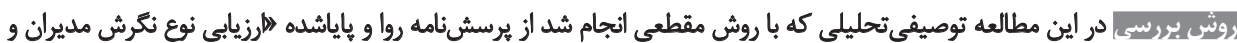

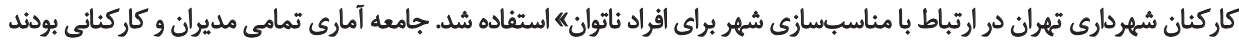

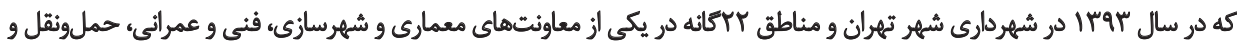

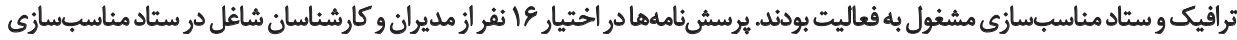

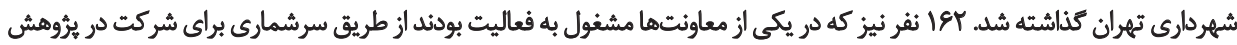

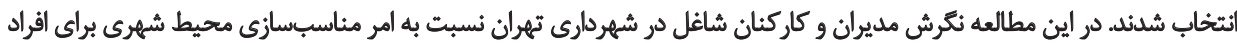

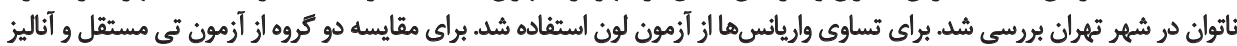

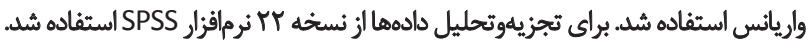

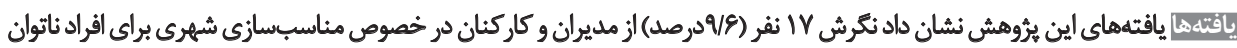

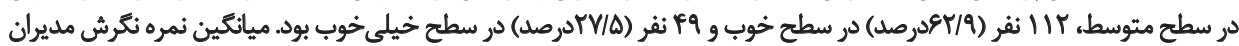

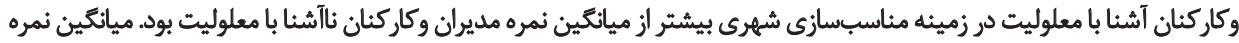

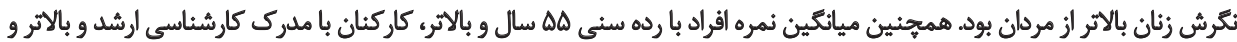

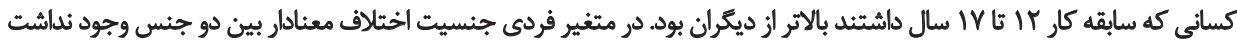

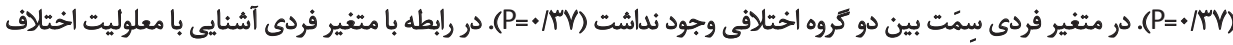

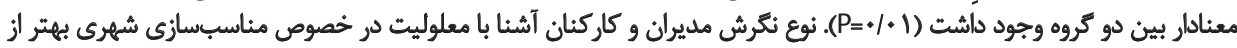

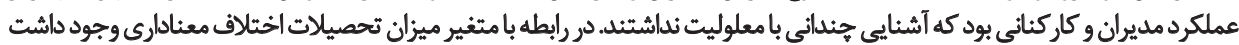

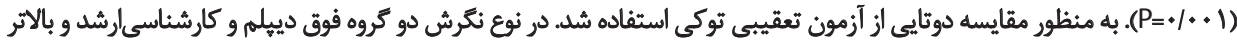

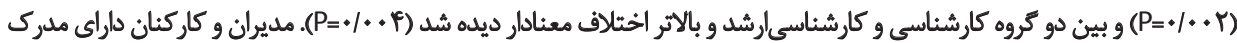

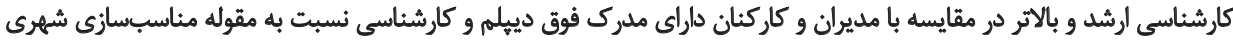
نكرش بهترى داشتئد.

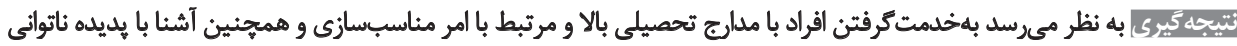

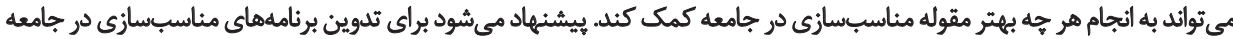

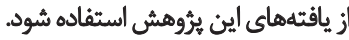

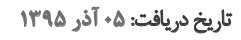

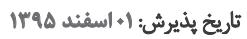

كليدوأوهها: نتكرشسنجى، كاركنان، مثاسبسازي، أفراد داراى ناتوانى، شهردارى أرادي 
اين مشكل بايد ساختارهاي عينى زندكى براي افراد داراي ناتوانى

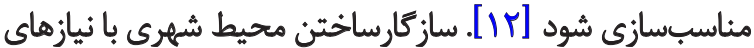

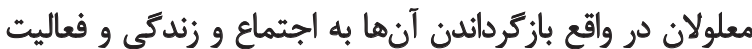

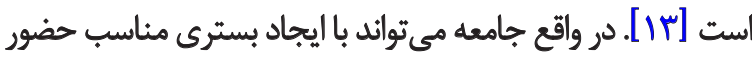

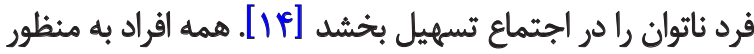

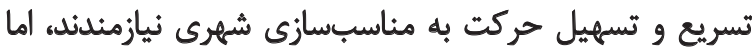

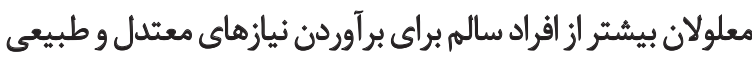

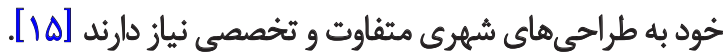
با توجه به اهميت مشاركت حداكثرى سرمايههاى انسانى

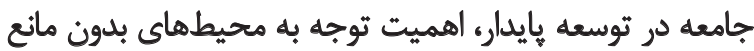

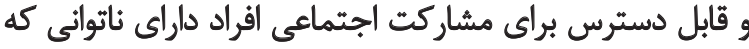

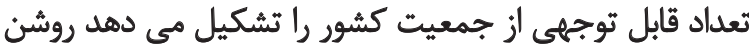

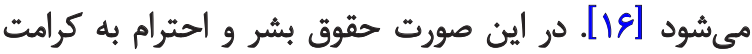

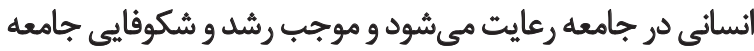

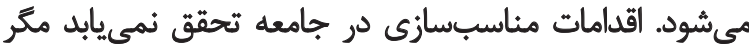

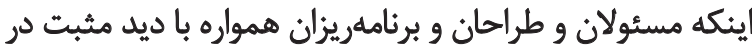

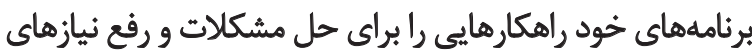

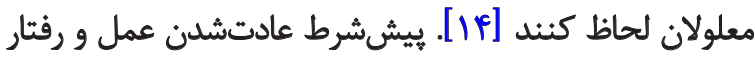

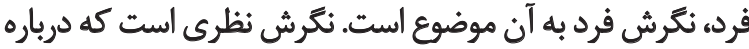

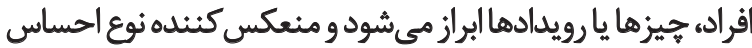

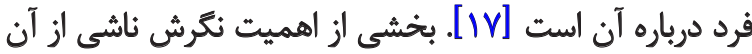

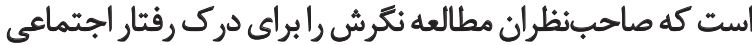

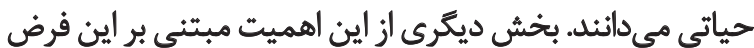

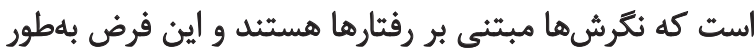

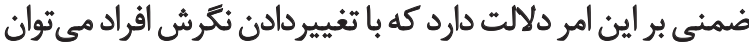

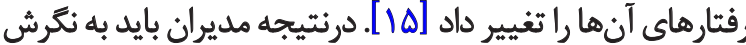

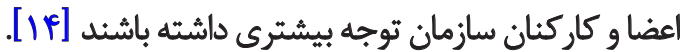

در كشورهاى درحالتوسعه كه أفراد داراى ناتوانى بيشترى

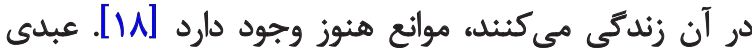

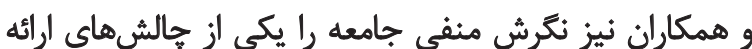

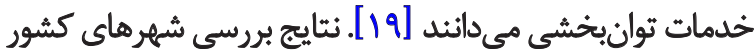

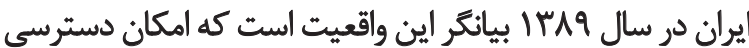

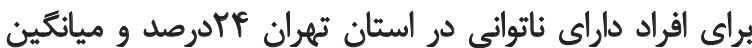

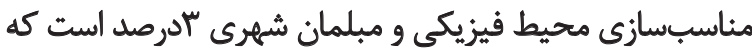

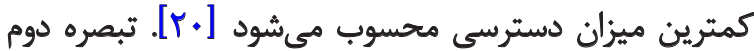

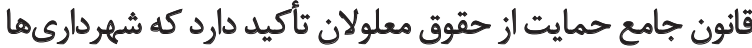

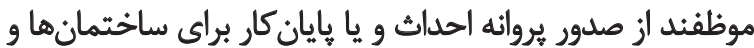

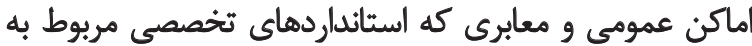

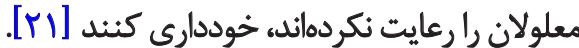
باوجود تمام اين تلاشهاو قوانين الزامآور ديكر مبنى بر وظيفه

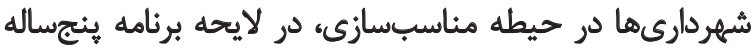

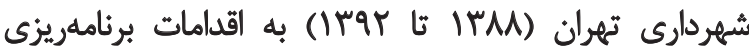
شهردارى تهران در زمينه مناسبسازى فضاهاى شهرى بران براى
مقلمه

طبق گَزارش سازمان بهداشت جهانى در سال II إك، در حال

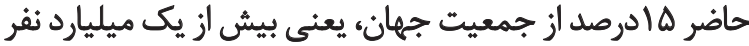

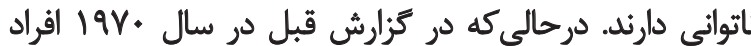

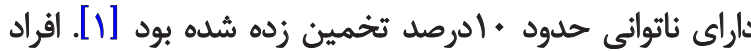

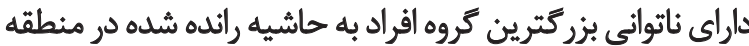

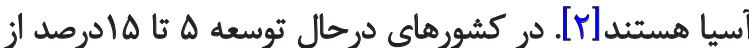

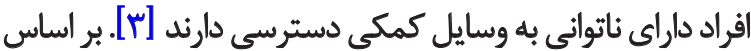

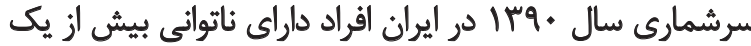

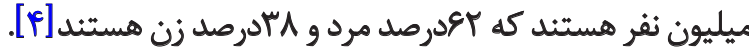

مفهوم برابرسازى فرصتها يكى مفهوم كليدى است. بر اساس

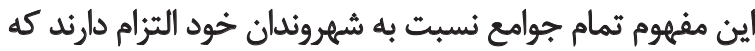

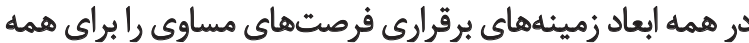

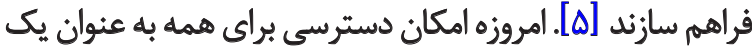

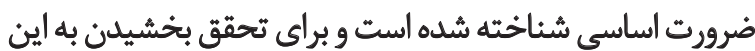

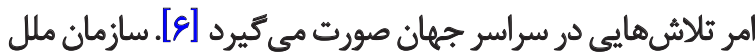

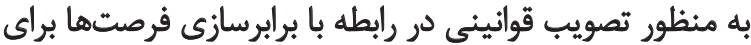

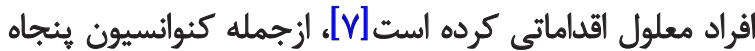

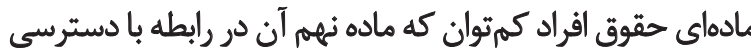

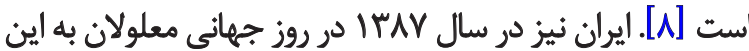

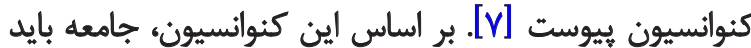

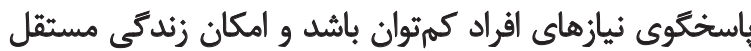

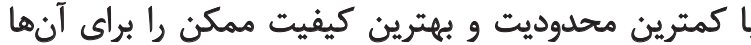

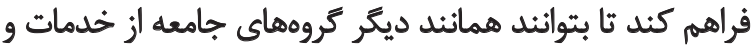

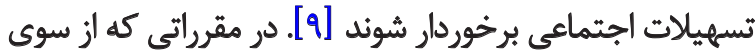

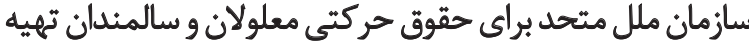

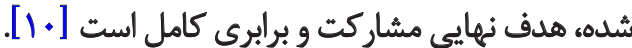
مسائل و مشكلات افراد داراى ناتوانى رامى مثوان در دو حالت

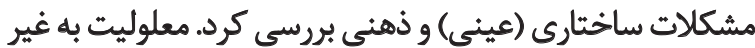

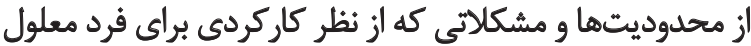

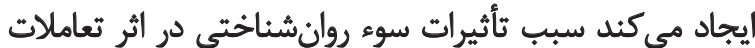

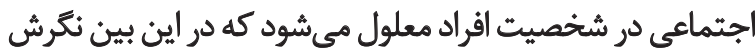

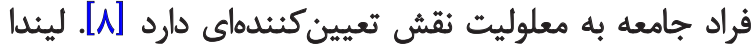

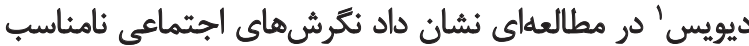

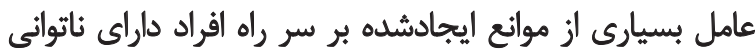

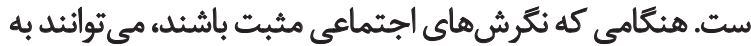

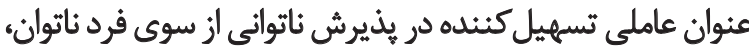

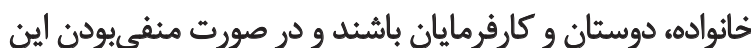

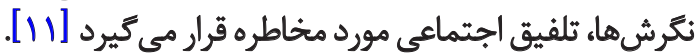
نكرش تبعيضآميز و بيشداورى جامعه نسبت به افراد داراى

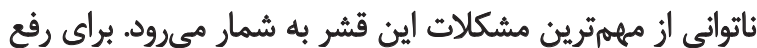

1. Linda Davis 
ييرامون دانشجويان ناتوان يرداختند. قابليت رقابت با دانشجويان

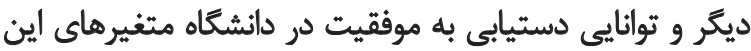

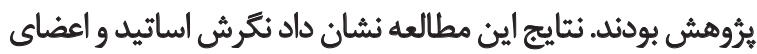

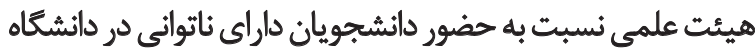

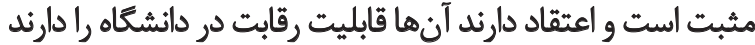

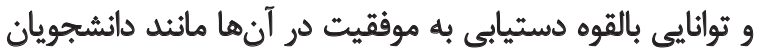

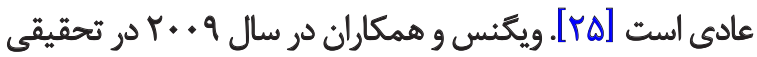

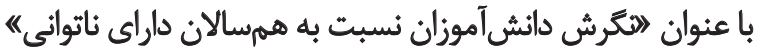

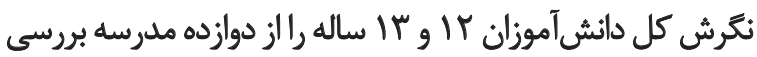

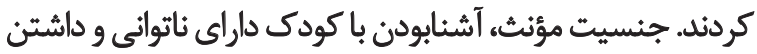

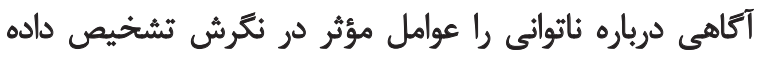

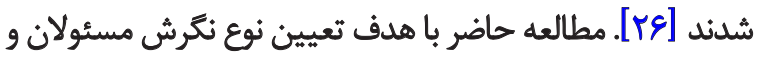

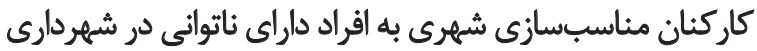

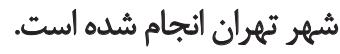

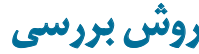

اين مطالعه توصيفىتحليلى با روش مقطعى انجام شد. جامعه

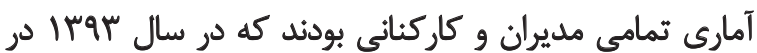

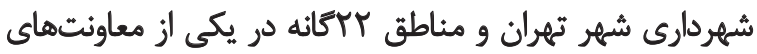

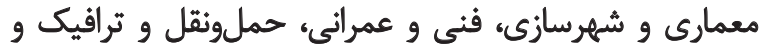

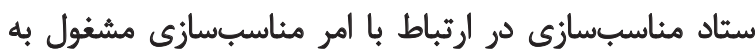

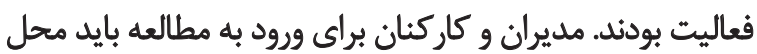

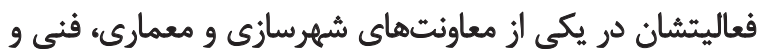

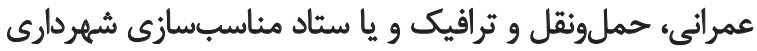

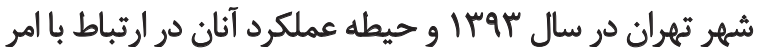

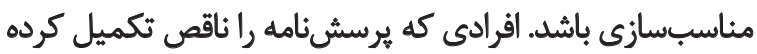

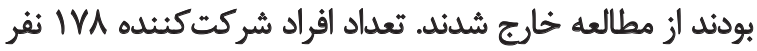

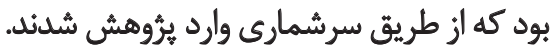

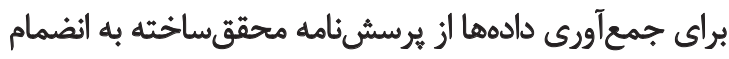

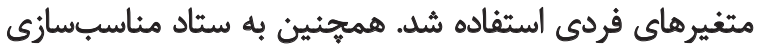

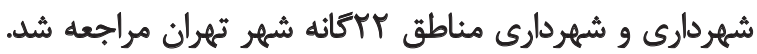

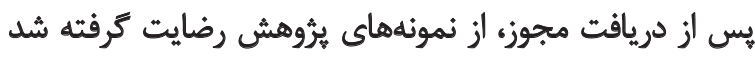

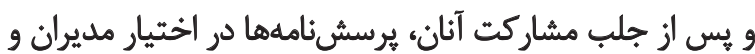

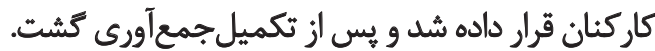

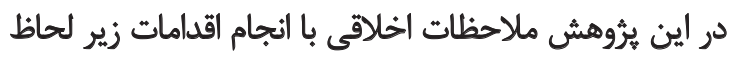

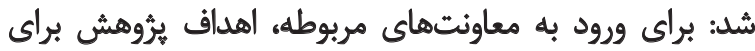

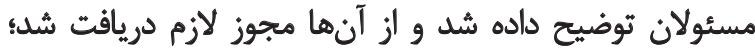

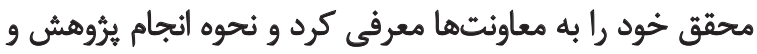

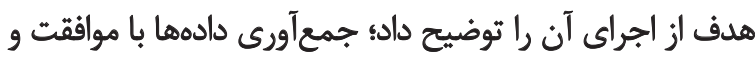

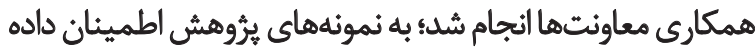

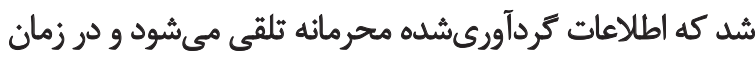

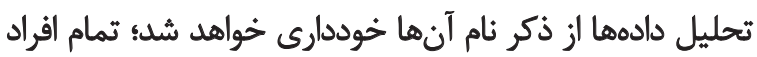

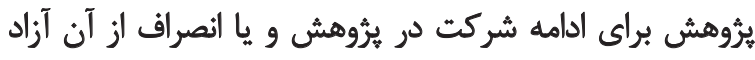

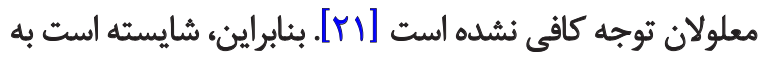

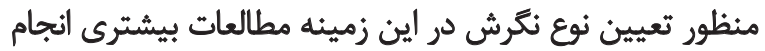

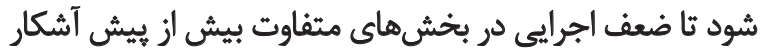
شود و مسئولان را به جستوجي در بخش به دنبال راهكار وادار كند. در ايران مسئولان و كارشناسان شهردارى تحقيقات بسيار

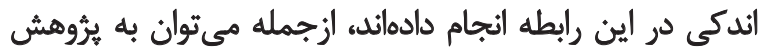

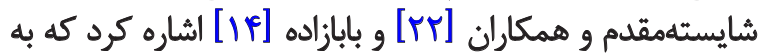

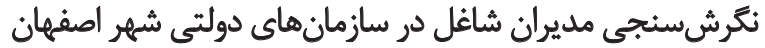

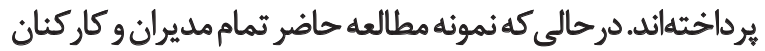

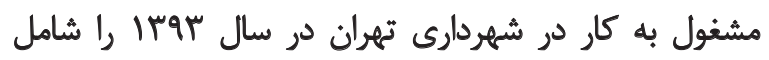

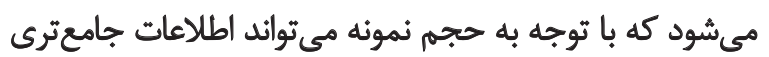

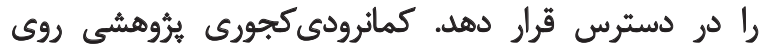

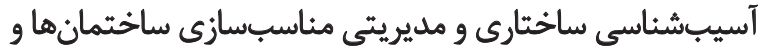

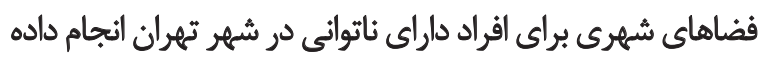

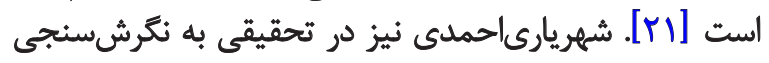

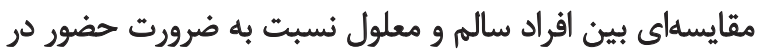

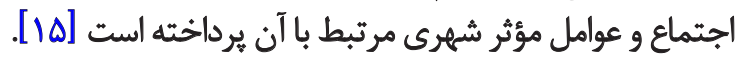
عبدى و همكاران (19 (Y) در مطالعهايى به بررسى موانع ارائه

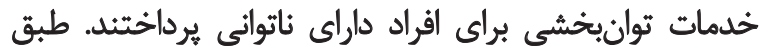

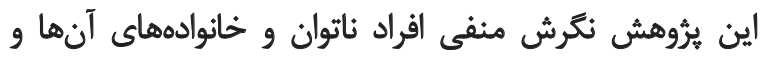

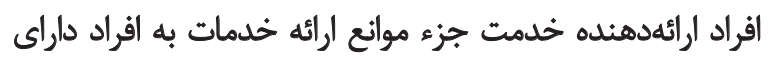

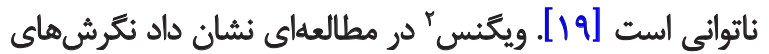

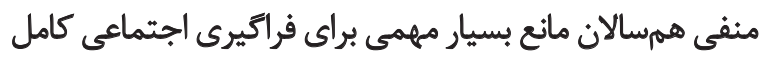

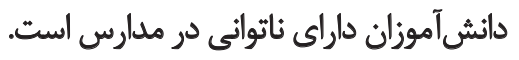

يس از تعيين نكرش كروهوهاى مختلف مي توان برنامهنهاى

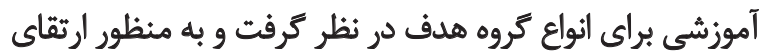

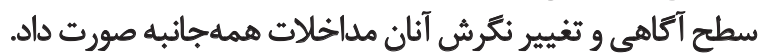

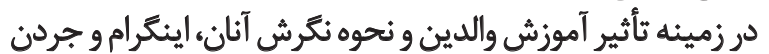

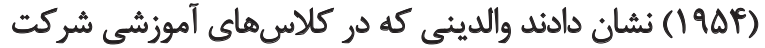

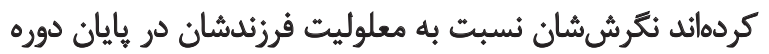

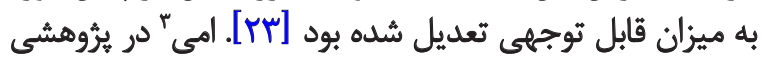

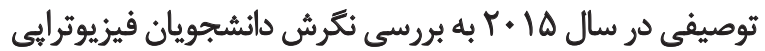

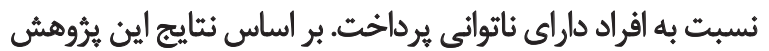

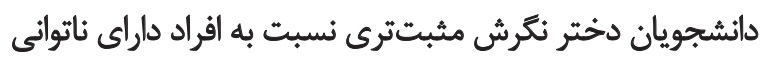

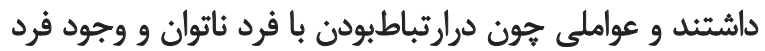

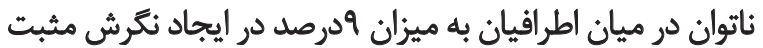

تأثير كذار است [rF]

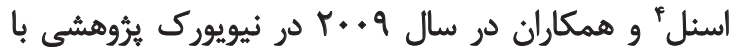

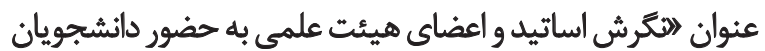

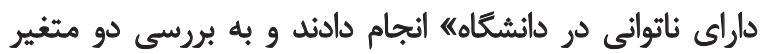


متغيرهاي فردى جنسيت، سمَت و آشنايي با معلوليت را نشان

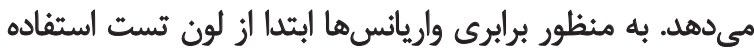
شد. سيس از آزمون تى مستقل براي مقايسه دو كروه استفاده شد. جدول شماره Y نشان مى دهد در متغير فردى جنسيت بين

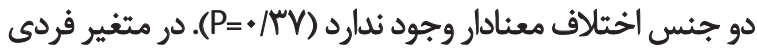

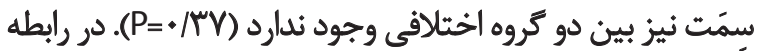

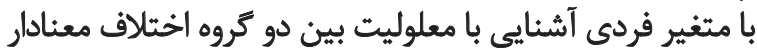

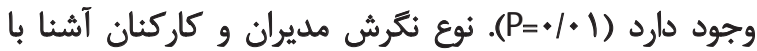
معلوليت در خصوص مناسبسازى شهرى بهتر از عملكرد مدير إيران و كاركنانى بود كه با معلوليت آشنايى نداشتيند.

جدول شماره ب عملكرد مديران و كاركنان شهردارى را با فيا متغيرهاى فردى ميزان تحصيلات، محل فعاليت و سابقه كارئ كارى

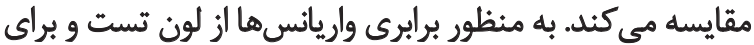

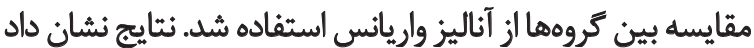

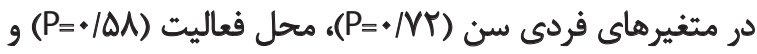

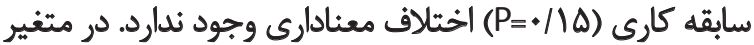

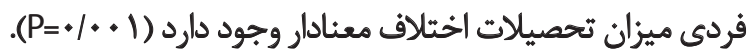
در رابطه با متغير فردى ميزان تحصيلات كه واريانسها

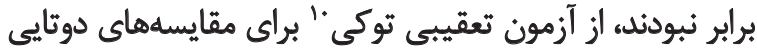

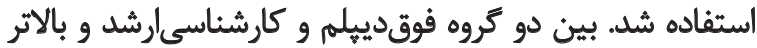

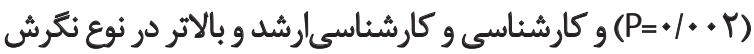

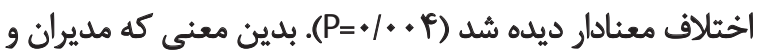

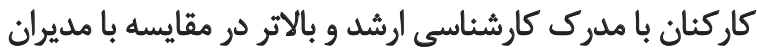

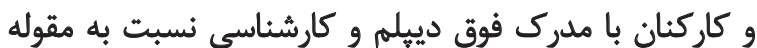
مناسبسازي نكَرش بهترى داشتند.

بحث

مطالعه حاضر با هدف تعيين نوع نكرش مديران و و كاركنان

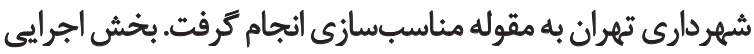

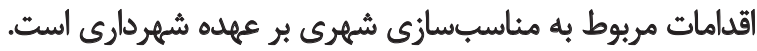

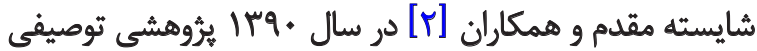

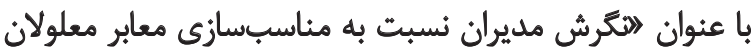

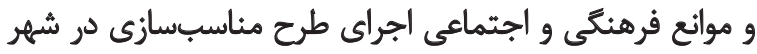

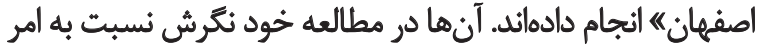

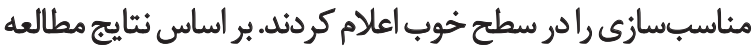

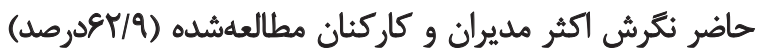

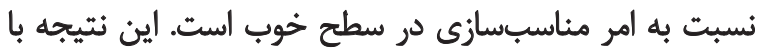

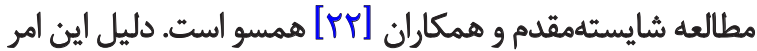

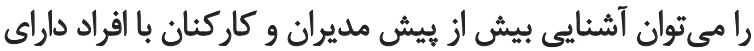
ناتوائى و كسب اطلاع از تأثير مناسبسازي دي در زئدئى آنان دائست.

10. Tukey Test
بودند؛ به افراد شركت كننده در يرّوهش توضيح داده شد كه در

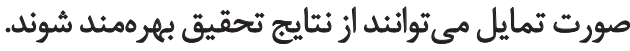

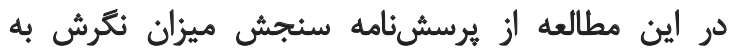

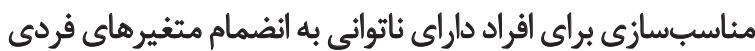

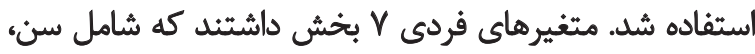

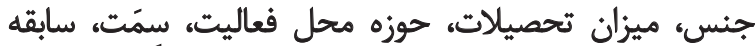

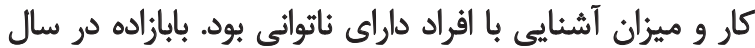

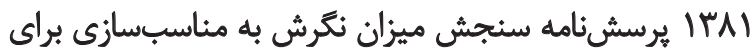

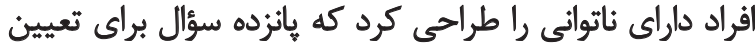

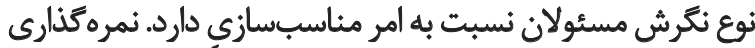

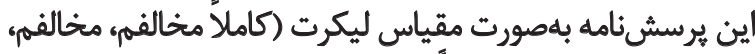

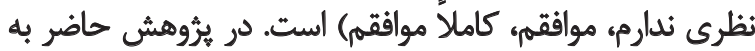
دليل ايجاد تغييرات در برخى از سؤالها، طبق نافيق نظر تيم تحقيق روايى و يايايى آن مجددا إنجام شد.

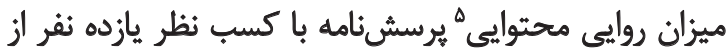

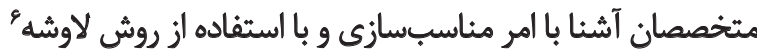

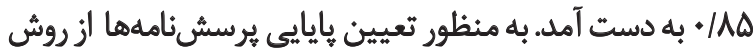

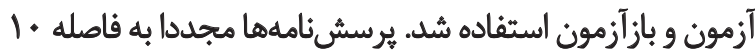

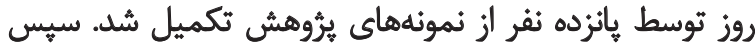

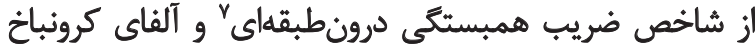

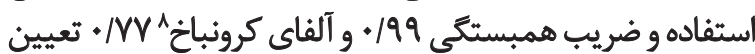

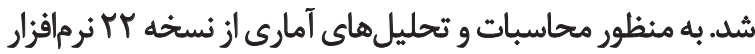

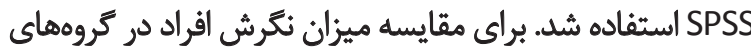

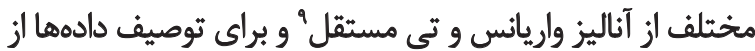

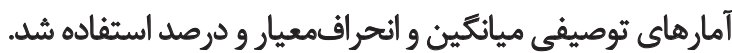

Latöl

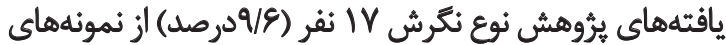

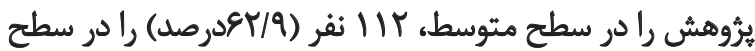

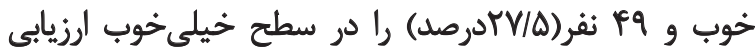

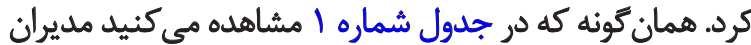

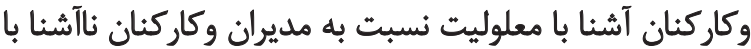

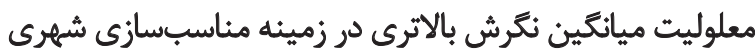

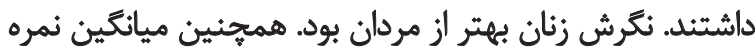

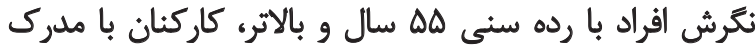

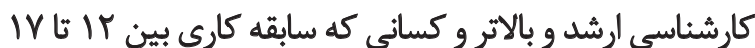

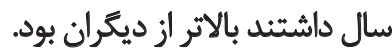

جدول شماره Y مقايسه نكرش مديران و كاركنان شهردارى با

5. Content validity

6. Lawshe's Technique

7. Intra-class Correlation Coefficients (ICC)

8. Cronbach's alpha

9. Independent t-test 


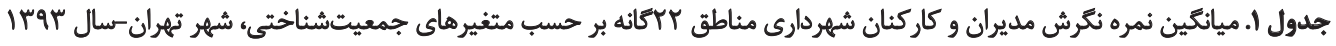

\begin{tabular}{|c|c|c|c|c|c|}
\hline انحراق معيار & مياكگين & درصد & تعداد & توصيف & مثغير \\
\hline $8 / 14$ & $8 . / 4$ & $\Delta q$ & $1+0$ & 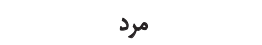 & \multirow[b]{2}{*}{ جنس ج ج ج } \\
\hline$V / V A$ & $81 / T V$ & it & $n^{r}$ & زن & \\
\hline $9 / 10$ & $\Delta 9 / 19$ & $r \cdot / \Lambda$ & rv & هدير & \multirow{3}{*}{ سمت } \\
\hline & & & & & \\
\hline V/*r & $81 / .4$ & $v a r$ & $\mid+1$ & 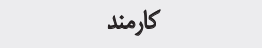 & \\
\hline$g / 4^{*}$ & $81 / 49$ & AV/ & & 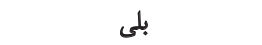 & \multirow{3}{*}{ آشنايى با هعلوليت } \\
\hline & & & & & \\
\hline$V / 19$ & $\Delta N M$ & $r / 9$ & rq & 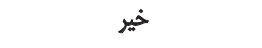 & \\
\hline$V / \backslash u$ & $\Delta Q / .$. & $g / r$ & 11 & r r- & \multirow{6}{*}{ سن } \\
\hline V/er & $81 / \pi$ & pels & AN & Tlr-rV & \\
\hline $9 / 4$. & $8.1 W$ & $M / N$ & $M$ & & \\
\hline 91.9 & $\Delta \mathrm{Q} / \mathrm{QA}$ & $1 \% / \Delta$ & re & WV-P & \\
\hline e/TA & $9 . / \pi$ & $\Delta / 1$ & 9 & & \\
\hline$\Delta / \Delta$. & $9 \pi / 4 T$ & $r / 9$ & $\checkmark$ & هـ سال و بالاتر & \\
\hline$s / \mathrm{VA}$ & $\Delta \Delta / \ldots$ & $T / T$ & $f$ & دييلم & \multirow{5}{*}{ ميزأن تحصيلات } \\
\hline $8 / 9 \%$ & $\Delta S / 1 Q$ & $\checkmark / T^{\circ}$ & ir & فوق دييلم & \\
\hline & & & & & \\
\hline$V / \backslash \Delta$ & $\Delta Q / 8 \Delta$ & $\mathrm{FV} / \mathrm{h}$ & Na & كارشناسى & \\
\hline$\Delta / \%$ & $8 \pi / M$ & $r T / \mathrm{V}$ & ve & كارشناسى ارشد و بالاتر & \\
\hline g/rג & $g / \Delta F$ & 9 & 18 & ستاد مثاسبسازى & \multirow{4}{*}{ هحل فعاليت } \\
\hline E/A9 & 8.19 & $r T / N$ & $v e$ & هماونت معمارى و شهرسازى & \\
\hline V/Tr & $\Delta Q / Q$ & rt & $\Delta Y$ & معاونت فُنى و عمراثى & \\
\hline e/rA & $81 / .4$ & $1 \% / \pi$ & rq & معاونت حمل ونقل و ترافيى & \\
\hline$g / M$ & s//Ar & $r \cdot / q$ & $\Delta \infty$ & 1-ه سال & \multirow{5}{*}{ سابقه كارى } \\
\hline$V / r q$ & $\Delta q / T V$ & $r q / q$ & $n$ & ع-811 سال & \\
\hline$\Delta / T Y$ & gT/FQ & $1 \% / 8$ & re & YIV-IV سال & \\
\hline$\Delta / I r$ & $9 \cdot / M$ & 9 & 18 & גו-1"r سال & \\
\hline$V / M r$ & $8 \cdot 11$. & $\Delta / F$ & 1. & זٓ سال و بالاتر & \\
\hline
\end{tabular}

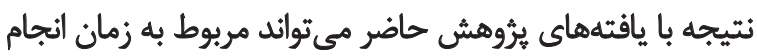

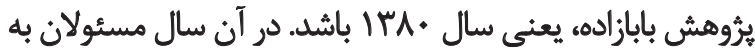

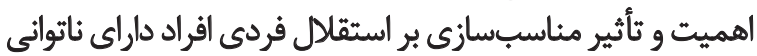

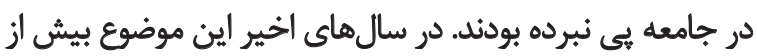

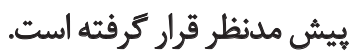

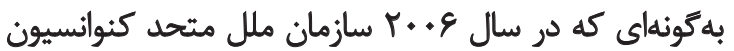

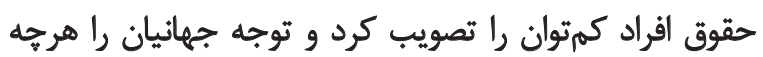

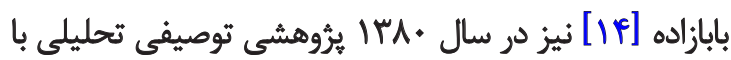

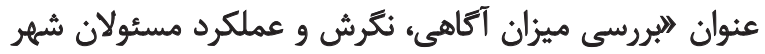

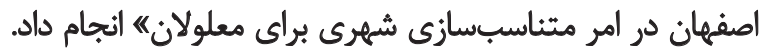

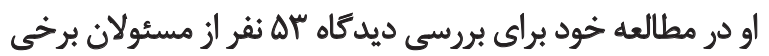

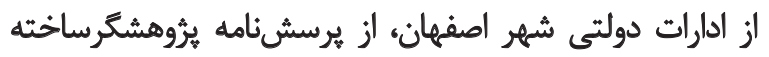

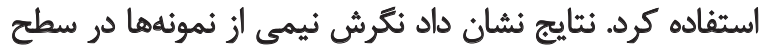

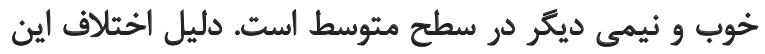




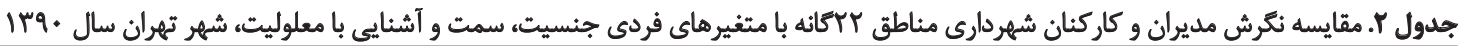

\begin{tabular}{|c|c|c|c|}
\hline \multicolumn{3}{|c|}{ T-Test } & \multirow{2}{*}{ ويرٔكى } \\
\hline P-Value & درجه آزادى & مقدار t & \\
\hline$\cdot \pi r$ & $15+/ 99$ &.$- / 19$ & جُسيتيت \\
\hline.$/ r V$ & ive & $-+/ 9$ & سمت \\
\hline .1 .1 & ive & $r / \Delta A$ & آشنايعي با هعلوليت \\
\hline
\end{tabular}

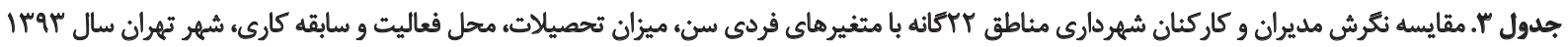

\begin{tabular}{|c|c|c|c|c|c|}
\hline \multicolumn{4}{|c|}{ آزمون مقايسه ميانكين هيثد جامعه } & \multirow{2}{*}{\multicolumn{2}{|c|}{ ويزٔى }} \\
\hline P-Value & $\mathbf{F}$ & ميانكين توان دوم كروهها & درجه آزادى & & \\
\hline \multirow{3}{*}{$\cdot M$} & \multirow{3}{*}{.$/ 0 Y$} & TV/TE & $\Delta$ & بين كروهى & \multirow{3}{*}{ سن } \\
\hline & & & & & \\
\hline & & $P V / \Delta q$ & in & درون كروهى & \\
\hline \multirow[b]{2}{*}{$<* 1 \cdot .1$} & \multirow[b]{2}{*}{$V / \Delta$} & $M+1 \circ F$ & r & بين Sروهى & \multirow[b]{2}{*}{ ميزان تحصيلات } \\
\hline & & & $1 n^{\circ}$ & sون & \\
\hline \multirow{2}{*}{.$/ 14 A$} & \multirow{2}{*}{.180} & ryloor & r & بين كروهى & \multirow{2}{*}{ محل فعاليت } \\
\hline & & $F V / \%$. & $i n$ & درون كروهي & \\
\hline \multirow{2}{*}{.110} & \multirow[b]{2}{*}{$M$} & ra/se & $f$ & بين كروهى & \multirow{2}{*}{ سابقه كارى } \\
\hline & & rems & in & درون كروهى & \\
\hline
\end{tabular}

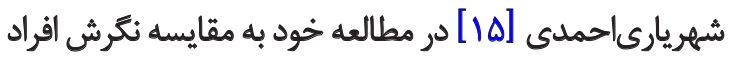

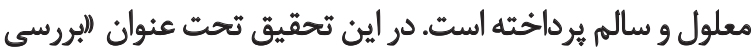

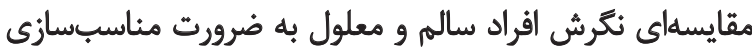

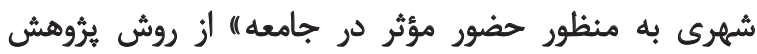

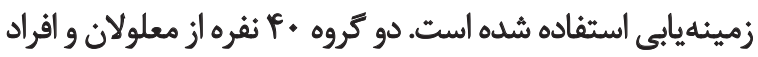

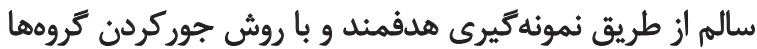

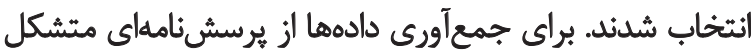

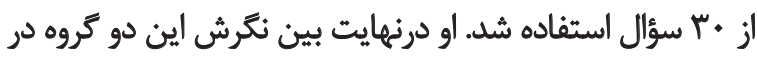

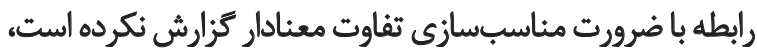

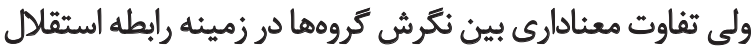
فردى با ساختار فيزيكى مناسب شهر كزارش كرئ كرده است.

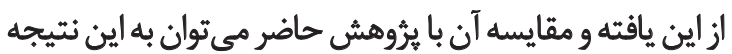

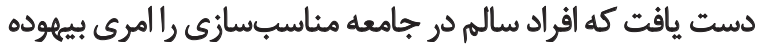

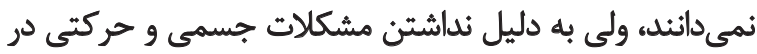

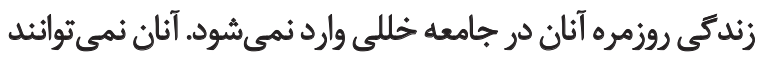

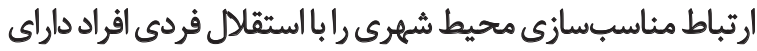

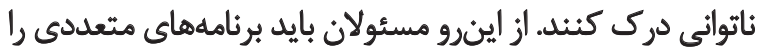

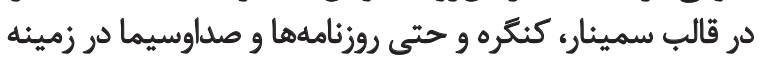
معرفى مشكلات افراد داراى ناتوانى و راههاى رفع اين مشكاملات

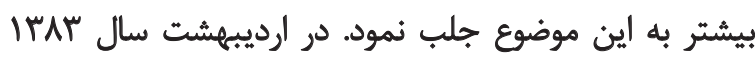

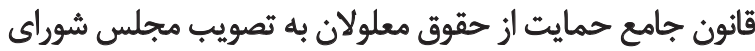

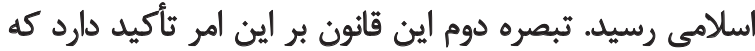

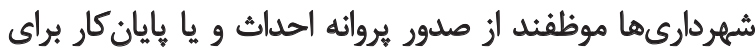

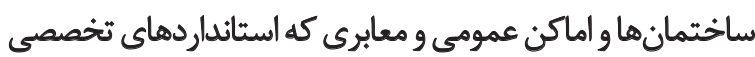

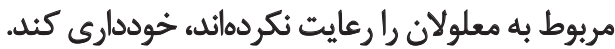
ازطرفى مى توان به نقش تأثير كذار ستاد مناسبسازي فضاهاي

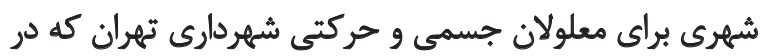

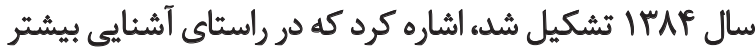

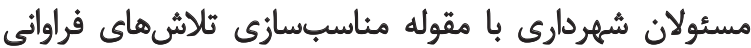

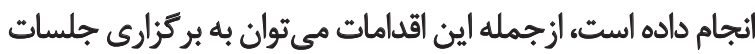

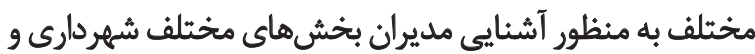

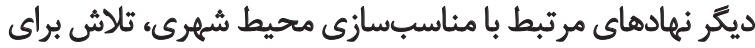

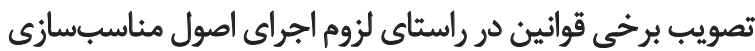

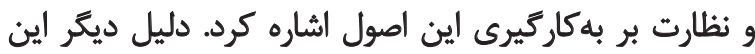

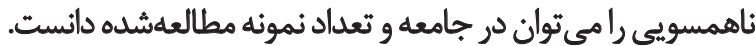

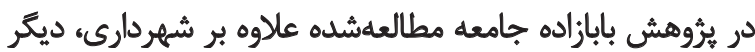
ادارات دولتى شهر اصفهان و با تعداد نمونه باهه نفر بوده است. 
مسئولان بامدرى كارشناسى ارشد نسبت به افراد با مدارك يايينتر

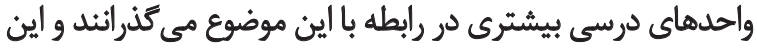

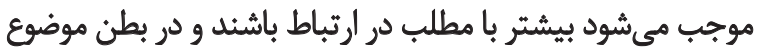

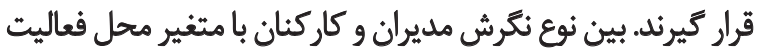

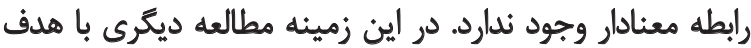

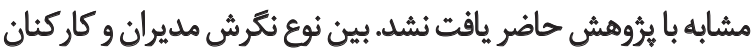

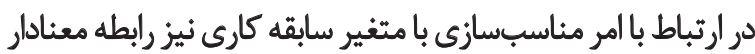

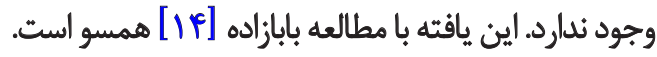

\section{نتيجلمكيرى}

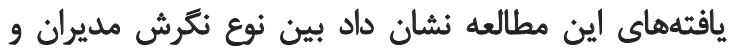

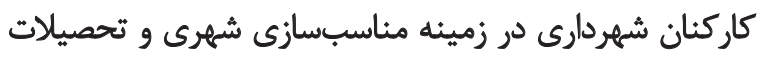

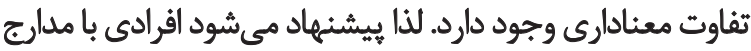

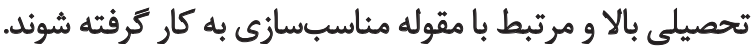

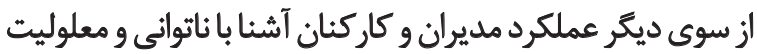
در سطح بهترى نسبت به ديكران قرار داشت.

شايسته است هنكام جذب نيرو، قوانينى در نظر كرفته شود كه

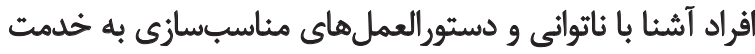

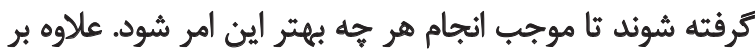

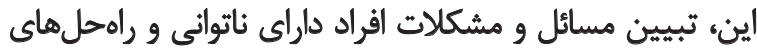

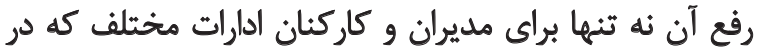

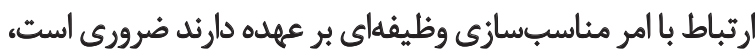

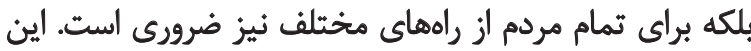

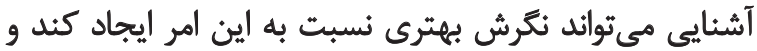

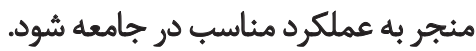

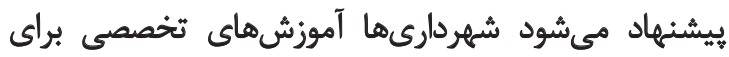

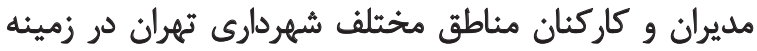

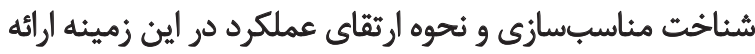

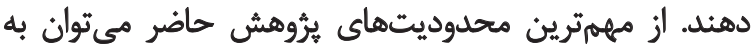

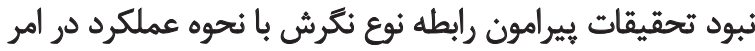

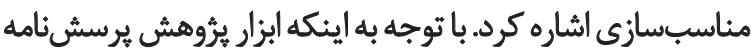

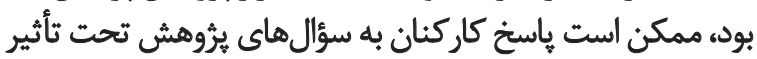
برخى ملاحظات الدارى و شغلى قرار كرفته باشند.

$$
\text { تشكير و قدرواني }
$$

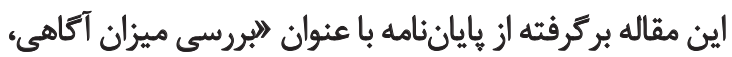

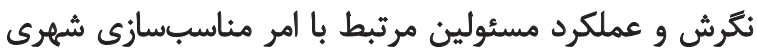

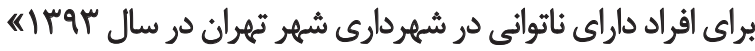

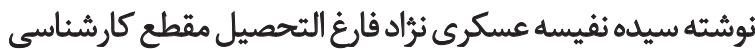
ارشد رشته مديريت توانبخشى است.
كه از جمله آنها مناسبسازى محيط شهرى است تدوين كنيند

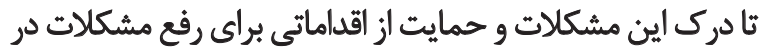

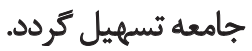

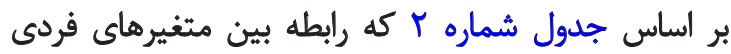

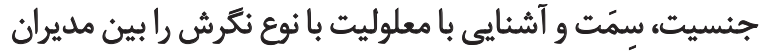

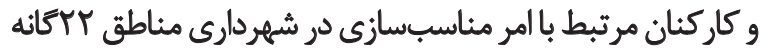

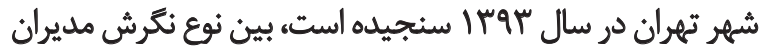

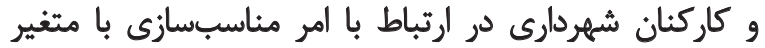

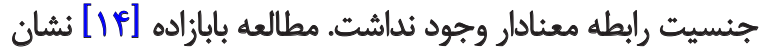

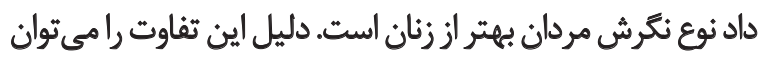

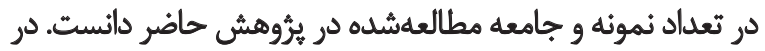

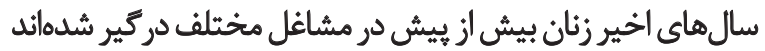

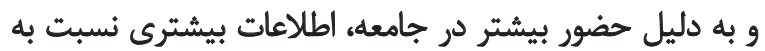

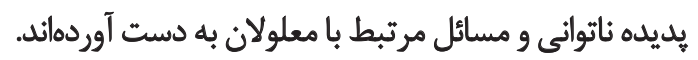

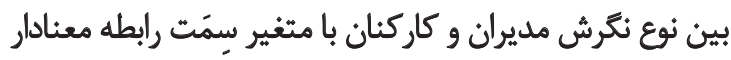

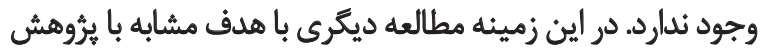

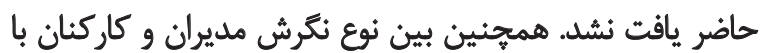

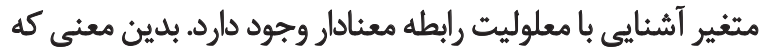

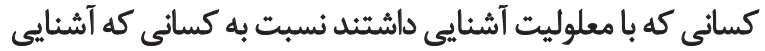

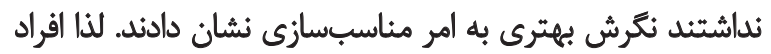

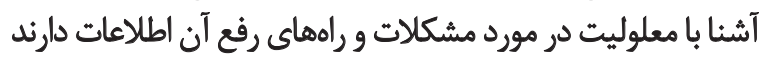

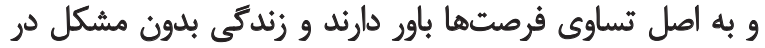

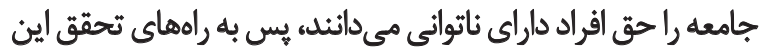

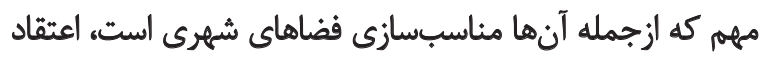

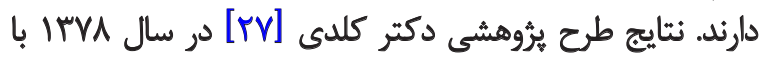

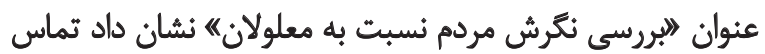

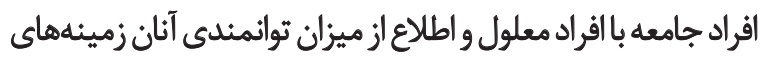

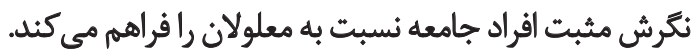
بر اساس جدول شماره ب بين نوع نترش مديران و كاركنان

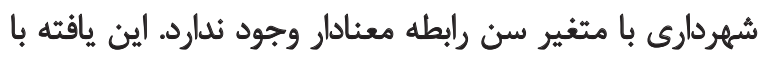

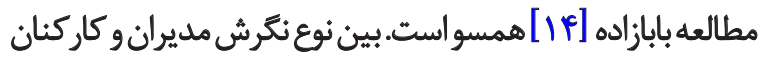

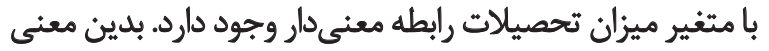

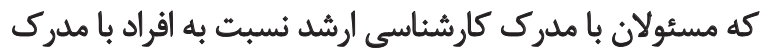

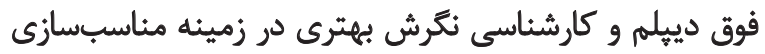

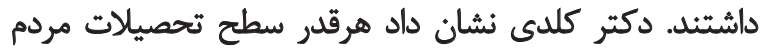

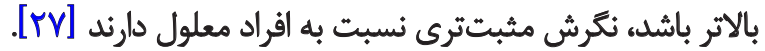

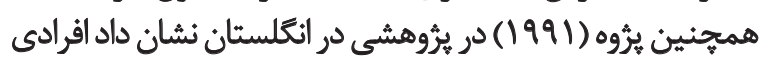

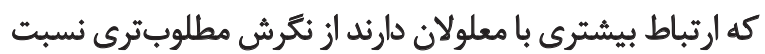

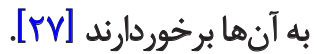

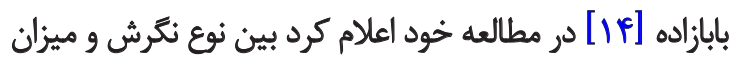

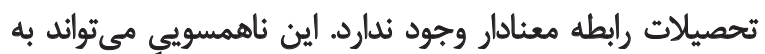

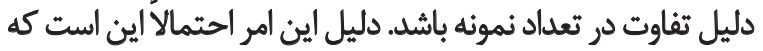




\section{References}

[1] World Health Organization. World report on disability. Geneva: World Health Organization; 2011.

[2] Abdi K, Arab M, Rashidian A, Kamali M, Khankeh HR, Khalajabadi Farahani F. Exploring barriers of the health system to rehabilitation services for people with disabilities in Iran: A qualitative study. Electronic Physician. 2015; 7(7):1476-85. doi: $10.19082 / 1476$

[3] World Health Organization. Assistive devices/technologies. Geneva: World Health Organization; 2010.

[4] Statistical Center of Iran. National population and housing census 2011: Selected findings. Tehran: Statistical Center of Iran; 2011.

[5] Kamali M. [People with disabilities (disabled) in the barrier-free cities (Persian)]. Paper presented at: The National Conference on Enabling the Urban Environment for the Disabled; 2007 February 3; Tehran, Iran.

[6] Ferneeuw S. Guidelines for planning a barrier-free environment: a practical manual to improve physical accessibility in Afghanistan [Internet]. 2005 [Cited 2005 March 24]. Available from: http:// www.asksource.info/resources/guidelines-planning-barrier-freeenvironment-practical-manual-improve-physical

[7] Moradi M, Baghestani A, Tafaroshi F, Raees Dana M. [Investigating the status of people with disabilities access to facilities and public spaces all over the country (Persian)]. Tehran: State Walfare Organization; 2010.

[8] United Nations. Convention on the rights of persons with disabilities. New York: United Nations; 2006

[9] Zaman-pour M, Hatami-Zadeh N, Bakhshi E. [Assistive technology needs assessment from adolescent students with hearing loss and their parents, Ahvaz City-2013 (Persian)]. Journal of Rehabilitation. 2015; 15(4):42-51.

[10] Nozari S. [The appropriate administrative structure for disabled people (Persian)]. Paper presented at: The First National Conference on Adjusting the Urban Environment; 2005 February 14; Tehran, Iran.

[11] Davis L. Disabilities in the workplace: recruitment, accommodation, and retention. AAOHN Journal. 2005; 53(7):306-12. PMID: 16097104

[12] International Labour Office. Achieving equal employment opportunitiesfor people with disabilities through legislation [Babaei Ahari M, Persian trans]. $1^{\text {st }}$ ed. Tehran: Technical Consulting Organization; 1994.

[13] Afsah Hoseini F. [Adjusting the urban furnitures (Persian)]. Paper presented at: Paper presented at: The First National Conference on Adjusting the Urban Environment; 2006 February 14; Tehran, Iran.

[14] BabaZadeh M. [Assessment of awareness, attitude and performance of responsibles related to the urban modification for handicapped in Isfahan (Persian)] [MSc. thesis]. Tehran: University of Social Welfare and Rehabilitation Sciences; 2001.

[15] Shahryari Ahmadi M. [Comparative study of the attitudes of healthy people and people with disabilities to the necessity of adjusting the city to participate effectively in society (Persian)]. Paper presented at: Paper presented at: The First National Conference on Adjusting the Urban Environment; 2006 February 14; Tehran, Iran.

[16] Borjian M, Joghataei B. [Adaptation of building standards in urban environments for disabled people (Persian)]. $1^{\text {st }}$ ed. Tehran: State Welfare Organization; 2009.

[17] Robbins S. Organizational behavior: concepts, controversies, applications. [Parsian A, Arabi SM, Persian trans]. $2^{\text {st }}$ ed. Tehran: Cultural Research Bureau; 2014.

[18] Bajlan S. [Checking the status of people with disabilities access to facilities and public spaces of Universities in Tehran (Persian)] [MSc, thesis]. Tehran: Iran University of Medical Sciences; 2008.

[19] Abdi K AM, Rashidian A, Kamali M, Khankeh HR, Farahani. $\mathrm{F}$, et al. Challenges in providing rehabilitation services for people with disabilities in Iran: A qualitative study. British Journal of Medicine \& Medical Research. 2016; 13(4):1-11. doi: 10.9734/ bjmmr/2016/23337

[20] State Walfare Organization. [Appropriate state of buildings and public places, the physical environment and urban furniture across the country (Persian)]. Tehran: State Walfare Organization; 2010 .

[21] Kamanroodikajuri M. [Structural pathology of management amd urban development: making appropriate Tehran spaces for the disabled (Persian)]. Urban Management. 2010; 8(25):99-114.

[22] Shayesteh Moqaddam M, Esmaeeli R, Noori A. [Adjusting the sidewalks for the people with disability and cultural barriers to its implementation (Persian)]. 2011 [Cited 2011 Aug 12]. Available from: https:// sociologyofiran.com/?p=1467

[23] Loaliyan Tamijani S. [Comparing the attitude of nurses and mambers of rehabilitation for handicapped in hospitals and rehabilitation centers in Tehran (Persian)] [MSc. thesis]. Tehran: University of Social Welfare and Rehabilitation Sciences; 2007.

[24] Yorke AM, Ruediger T, Voltenburg N. Doctor of physical therapy students' attitudes towards people with disabilities: A descriptive study. Disability and Rehabilitation. 2016; 39(1):91-7. doi: 10.3109/09638288.2016.1140830

[25] Linda H. Sniatecki JLS, Perry Holly B. Faculty attitudes and knowledge regarding college students with disabilities. Journal of Postsecondary Education and Disability. 2009; 28(3):259-75. doi: $10.1177 / 08857288050280010501$

[26] Schutzer K. Barriers and motivations to exercise in older adults. Preventive Medicine. 2004; 39(5):1056-61. doi: 10.1016/j. ypmed.2004.04.003

[27] Kaldi A. [Assessment of attitude of peoples about Handicapped in Zanjan (Persian)]. Tehran: University of Social Welfare and Rehabilitation Sciences; 2000 
